\title{
An Analysis of Helium Primordial Nucleosynthesis with a Variable Cosmological Coupling
}

\author{
F.G. Alvarenga, J.C. Fabrisł ${ }^{\dagger}$ S.V.B. Gonçalves $;$ and J.A.O. Marinho ${ }^{\S}$ \\ Departamento de Física, Universidade Federal do Espírito Santo, \\ CEP 29060-900, Vitória, Espírito Santo, Brazil
}

Received on 21 May, 2001

\begin{abstract}
The synthesis of helium in the early Universe depends on many input parameters, including the value of the gravitational coupling during the period when the nucleosynthesis takes place. We compute the primordial abundance of helium as function of the gravitational coupling, using a semi-analytical method, in order to track the influence of $G$ in the primordial nucleosynthesis. To be specific, we construct a cosmological model with varying $G$, using the Brans-Dicke theory. The greater the value of $G$ at nucleosynthesis period, the greater the predicted abundance of helium. Using the observational data for the abundance of primordial helium, constraints for the time variation of $G$ are established.
\end{abstract}

\section{Introduction}

The primordial nucleosynthesis is one of the most important achievements of the cosmological standard model $[1,2]$. Using the Einstein's equations, supposing a flat, isotropic and homogeneous space-time, considering a radiation dominated initial phase in the evolution of the Universe, it has been shown that it is possible, assuming initially an equal distribution of protons and neutrons, to obtain the primordial production of helium, leading to an abundance of this element of about $24 \%$ of the mass of the Universe. The observational data indicates an abundance of helium of $Y_{4}^{o b s}=0.241 \pm 0.002[3]$ in mass fraction. Deuterium and lithium, as well as ${ }^{3} \mathrm{He}$, are also produced. However, heavier elements can not be produced due to the absence of stable nuclei with $A=5$ and 8; heavier nuclei are produced later in the stars. Taking the ratio of baryons to photons $\eta$, as a free parameter, the predicted abundances agree with observations with a precision of some percents. For the moment, this is the earliest test of the standard model, giving confidence that the Universe followed, in general lines, the evolution predicted by the Hot Big Bang model up to 1 second of age. It is possible that the analysis of the anisotropy of cosmic background radiation may lead to tests concerning earliest moments. But, this remains just a possibility, even if the results are becoming more and more consistents.

The primordial nucleosynthesis, however, faces some controverses. As an example, it is considered

\footnotetext{
*e-mail: flavio@cce.ufes.br

†e-mail: fabris@cce.ufes.br

‡e-mail: sergio@cce.ufes.br

$\S$ e-mail: admarinho@hotail.com
}

now that lithium can also be produced in the stars, and the primordial abundance of this element could be smaller than it is assumed today [4]. Moreover, recent measurements of the second peak of the spectrum of the anisotropy of the cosmic background radiation, made by the BOOMERANG and MAXIMA atmospheric balloons programs, indicate a density of baryons in the Universe that agrees only marginally with that necessary to have the correct primordial production of light elements. In fact the nucleosynthesis requires $\Omega_{b} h^{2}=0.019 \pm 0.002$ [5], while the measurents of CMB anisotropy indicates $\Omega_{b} h^{2}=0.032 \pm 0.005$ [6]. However, there are claims that this discrepancy is less important than indicated by those analysis [7].

Even if the primordial nucleosynthesis remains one of the most impressive test of the standard model, it deserves yet more investigations. In particular, the primordial nucleosynthesis may be an arena to test the values of some input parameters. For example, the number of neutrino's families influence the primordial production of light elements. Hence, the primordial abundance of light nuclei permits to test if there are more than three families of leptons and quarks.

In the present work, another effect on the primordial nucleosynthesis will be exploited. It will be verified how the value of the gravitational coupling affects the primordial production of light elements. Hence, the primordial nucleosynthesis will be taken as another way to verify if the gravitational coupling varies with time. Studies in this sense have already been made by using 
computational codes [1] or semi-analytical methods as it will be used here, but in different context $[8,9]$. We will work out a specific case: the Brans-Dicke theory, which is the prototype of a relativistic gravity theory with varying $G$, will be considered.

The Brans-Dicke theory has an interesting connection with the low energy string action, this being another reason to study it. In the dust phase of the evolution of the Universe, power law type solution of the Brans-Dicke theory exhibits a decreasing gravitational coupling. This solution will be matched with the radiative solution, which in its simplest version coincides with that of the standard model. In this way the value of the $G_{R}$ at the moment of the nucleosynthesis will be connected to its value today, $G_{0}$. The value of $G_{R}$ depends on two input parameters: the ratio of the densities of radiation and baryonic matter today; the BransDicke coupling parameter $\omega$. It will be shown that the greater the value of $G$ at the moment of the nucleosynthesis, the greater the abundance of primordial helium.

The precise calculation of the primordial abundance of light elements is a very hard task. It implies to use many numerical codes in order to evaluate the transmutation process involving protons and neutrons to obtain the final ratio between these nucleons, which determines the final abundance of helium. In order to have very precise predictions, radiative corrections to the rate of the reactions must be taken into account. In the present work we will adopt the semi-analytical method developed in [10]. In this method the effects of the Fermi-Dirac statistic are neglected. This leads to a disagreement with respect to the precise calculation of the order of some percents. Since, the interest in this work it is to track the influence of a varying $G$ in the calculation of the primordial abundance of light elements, it is interesting to sacrify somehow the precison in favour of analytical expressions where the searched effects can be more easily tracked.

This paper is organized as follows. In section II, the Brans-Dicke cosmological model is developed, including the matching conditions. The relation between $G_{R}$ and $G_{0}$ is established. In section III, the computation method presented in [10] is outlined, stressing the rôle played by $G$. In section IV, the primordial abundance for a varying $G$ is obtained, in terms of the Brans-Dicke parameter $\omega$. In section $\mathrm{V}$ the conclusions are presented. Some considerations of how to reconcile a higher value for baryonic density, predicted by CMB anisotropy, with that one necessary to have abundance of primordial elements in agreement with observations, using a varying $G$ model, are sketched.

\section{The cosmological scenario}

The Brans-Dicke theory incorporates the space-time variation of the gravitational coupling in a relativistic theory of gravity [11]. In order to do so, it couples a scalar field non-minimally to gravity. Its Lagrangian reads,

$$
L=\sqrt{-g}\left[\phi R-\omega \frac{\phi_{; \rho} \phi^{; \rho}}{\phi}\right]+L_{m}
$$

where $R$ is the Ricci scalar, $\phi$ is a scalar field connected with the gravitational coupling, $\omega$ is the Brans-Dicke parameter and $L_{m}$ is the Lagrangian of matter, which will be taken as a barotropic perfect fluid with an equation of state $p=\alpha \rho,-1 \leq \alpha \leq 1$. General Relativity, and consequently a constant gravitational coupling, is recovered when $\omega \rightarrow \infty$. Using the flat FriedmannRobertson-Walker metric, the equations of motion read,

$$
\begin{aligned}
3\left(\frac{\dot{a}}{a}\right)^{2} & =\frac{8 \pi \rho}{\phi}+\frac{\omega}{2}\left(\frac{\dot{\phi}}{\phi}\right)^{2}-3 \frac{\dot{a}}{a} \frac{\dot{\phi}}{\phi}, \\
\ddot{\phi}+3 \frac{\dot{a}}{a} \dot{\phi} & =\frac{8 \pi}{3+2 \omega}(\rho-3 p), \\
\dot{\rho}+3 \frac{\dot{a}}{a}(\rho+p) & =0 .
\end{aligned}
$$

There are two phases which interest us: the dust phase, $\alpha=0$, and the radiation phase $\alpha=1 / 3$. For these phases, the equations $(2,3,4)$ admit the following powerlaw type solutions

$$
\begin{aligned}
p & =0 \rightarrow a \propto t^{\frac{2+2 \omega}{4+3 \omega}}, \\
\phi & \propto t^{\frac{2}{4+3 \omega}}, \quad \rho_{m} \propto a^{-3} ; \\
p & =\frac{\rho}{3} \rightarrow a \propto t^{1 / 2}, \\
\phi & =\text { constant }, \quad \rho_{r} \propto a^{-4} .
\end{aligned}
$$

In the above expressions, $\rho_{m}$ and $\rho_{r}$ are the density for dust and radiation respectivelly. For the radiative phase, the power-law solution coincides with that of the standard model, implying that the gravitational coupling is constant. But, since during the dust dominated phase, the gravitational coupling varies with time, the value of the gravitational coupling at the moment of the nucleosynthesis is, in this model, different from that normally used. Since the general features of the BransDicke model for the radiative phase is the same as in the standard model, we may compute the primordial abundance of light elements with a minimal modification. More general solutions, of course, can be found. We will discuss later the general features of these general solutions and their implications for nucleosynthesis.

In order to extract precise predictions, the solutions for the dust and radiative phases, as well as their first derivatives, must be matched. The solutions are rewritten as

$$
\begin{aligned}
& a=a_{0}\left(t-t_{m}\right)^{\frac{2+2 \omega}{4+3 \omega}} \quad(p=0) \\
& a=b_{0}\left(t-t_{r}\right)^{1 / 2} \quad\left(p=\frac{\rho}{3}\right)
\end{aligned}
$$




$$
\rho_{m}=\frac{\rho_{m 0}}{a^{3}} \quad, \rho_{r}=\frac{\rho_{r 0}}{a^{4}},
$$

where $a_{0}, b_{0}, t_{m}, t_{r}$ are constants; $\rho_{m 0}$ and $\rho_{r 0}$ are the density for matter and radiation today. Remembering that the gravitational coupling is connected with the value of the scalar field by $G=\left(\frac{4+2 \omega}{3+2 \omega}\right) \frac{1}{\phi}[12]$, and performing the matching of the solutions we find the following relation between the value of the gravitational coupling today $G_{0}$ and the value of the gravitational coupling during the radiative phase $G_{R}$ :

$$
G_{R}=\left(\frac{\rho_{m 0}}{\rho_{r 0}}\right)^{\frac{1}{1+\omega}} G_{0}
$$

It is more convenient to work with Planck's unity. Natural unities are employed: $c=\hbar=1, G=1 / \sqrt{M_{P}}$, $M_{P}$ being the Planck's mass whose value today is $M_{P 0}=1.221 \times 10^{19} \mathrm{GeV}$. Hence,

$$
M_{P R}=\left(\frac{\rho_{r 0}}{\rho_{m 0}}\right)^{\frac{2}{1+\omega}} M_{P 0},
$$

where $M_{P R}$ is the value of the Planck's mass at the moment of the nucleosynthesis.

\section{The semi-analytical compu- tation of the primordial nu- cleosynthesis}

The computation of the nucleosynthesis process in the early Universe involves three main steps. Initially, at very high energies, the ratio of neutrons to protons is equal to one. As the Universe expands, the temperature drops, and reactions involving the neutrons, protons, neutrinos, electrons convert neutrons into protons. At same time, since the neutrons are free, and unstable, they decay also into protons. This process continues until the energy is low enough in order the neutrons to be captured forming deuterium, from which the helium is formed. The quantity of helium synthetised in this process depends essentially on the quantity of neutrons that have survived up to the moment they are captured to form deuterium. The neutrons that are later used to form other elements like lithium are neglected in the present computation; anyway the others light elements besides helium represent a very small fraction of the total mass. The detailed analysis of all these process is quite involved, requiring the use of numerical codes to evaluate the different transmutation process. However, semi-analytical expressions can be worked out if the Fermi-Dirac statistics is negletected. This has been done in [10], leading to values for the helium abundance with an error of some percents compared with the precise numerical calculation.

Since all the evaluation of helium abundance in the approach used in [10] is very lengthy, we will just summarize the main steps and the relevant quantities. For details, the reader is invited to address himself to that work. First we define the ratio of neutrons with respect to the total baryon number:

$$
X(T)=\frac{n_{n}(T)}{n_{n}(T)+n_{p}(T)}
$$

where $n_{n}(T)$ and $n_{p}(T)$ are the numbers of neutrons and protons, respectively, as functions of the temperature $T$. The main equation controling this quantity is

$$
\frac{d X(t)}{d t}=\lambda_{p n}(t)(1-X(t))-\lambda_{n p}(t) X(t)
$$

where $\lambda_{p n}$ and $\lambda_{n p}$ are the rates of conversion of protons into neutrons and neutrons into protons respectively. The main process concerned are

$$
\lambda_{n p}=\lambda\left(\nu+n \rightarrow p+e^{-}\right)+\lambda\left(e^{+}+n \rightarrow p+\bar{\nu}\right)+\lambda\left(n \rightarrow p+\bar{\nu}+e^{-}\right)
$$

As an example, the first one is given by

$$
\lambda\left(\nu+n \rightarrow p+e^{-}\right)=A \int_{0}^{\infty} d p_{\nu} p_{\nu}^{2} p_{e} E_{e}\left(1-f_{e}\right) f_{\nu}
$$

where $A$ is a coupling constant, the $p$ 's denote the momenta of each particle involved in the process, $E$ the energy and the $f$ 's represents the Fermi-Dirac statistics factor. The last process in (13) represents the neutron decay and it is not considered in a first evaluation.
Later, the final results will be corrected taking it into account.

In [10] the evaluation of the first two rates in (13) is simplified by approaching the Fermi-Dirac statistics by the Maxwell-Boltzmann one. This is justified by the fact the temperatures concerned at the moment these process take place are smaller than the energies of the particles. After a lengthy evaluation of all process, we end up with the following expression for the neutron 
abundance factor:

$$
X(y)=X_{e q}(y)+\int_{0}^{y} d y^{\prime} e^{y^{\prime}} X_{e q}^{2}\left(y^{\prime}\right) \exp \left[K(y)-K\left(y^{\prime}\right)\right]
$$

with the following definitions:

$$
\begin{aligned}
X_{e q} & =\frac{1}{1+e^{y}} \\
K(y) & =b\left[\left(\frac{4}{y^{3}}+\frac{3}{y^{2}}+\frac{1}{y}\right)+\left(\frac{4}{y^{3}}+\frac{1}{y^{2}}\right) e^{-y}\right], \\
b & =a\left[\frac{45}{4 \pi^{3} N}\right]^{1 / 2} \frac{M_{p}}{\tau \Delta m^{2}} \quad, \quad a=4 A \tau(\Delta m)^{5} \quad, \quad y=\frac{\Delta m}{T}
\end{aligned}
$$

where $\Delta m$ is the mass difference between neutrons and protons, $\Delta m=1.294 \mathrm{MeV}$. The fraction of neutrons to baryons at the end of all those process, $\bar{X}$, is obtained by making $y \rightarrow \infty, \bar{X}=X(y \rightarrow \infty)$.

As stated before, initially the neutron decay is neglected. To correct the final results due to it we must evaluate the time taken in the capture process forming the deuterium. This time is given, in principle, by evaluating the capture process until a temperature of the order of the deuterium binding energy $\epsilon_{D} \sim 2.225 \mathrm{MeV}$. However, lower energies (of order of $E \sim 0.1 \mathrm{MeV}$ ) must be considered due to the fact that the enormous number of photons implies that deuterium dissociation continues to occur even when $T_{\gamma}<\epsilon_{D}$. In peforming this analysis, we must take into account the evolution of the Universe, which in this case is reflected by the equation

$$
\frac{\dot{T}_{\gamma}}{T_{\gamma}}=-\left[\frac{8 \pi \rho}{3 M_{P}^{2}}\right]^{1 / 2}
$$

since during the radiative phase $a \propto 1 / T_{\gamma}, T_{\gamma}$ being the photon temperature, which is approximately equal to the neutrino temperature at the relevant temperature scales considered in this computation. Moreover, the energy density is given by

$$
\rho=N_{\text {eff }} \frac{\pi^{2}}{30} T_{\nu}^{4},
$$

where

$$
N_{e f f}=N_{\nu}+\left(\frac{11}{4}\right)^{4 / 3} N_{\gamma} \sim 13 .
$$

The final results imply that the time of capture is given by

$$
t_{c}=\left[\frac{45}{16 \pi^{3} N_{e f f}}\right]^{1 / 2}\left[\frac{4}{11}\right]^{2 / 3} \frac{M_{P}}{T_{\gamma 0}^{2}}+t_{0}
$$

The constant $t_{0}$ is

$$
\begin{aligned}
t_{0} & =\frac{11}{6 N_{e f f}}\left[\left(\frac{11}{4}\right)^{1 / 3}-1\right] t_{1}, \\
t_{1} & =\left[\frac{45}{16 \pi^{3} N_{e f f}}\right]^{1 / 2}\left(\frac{11}{4}\right)^{2 / 3} \frac{M_{P}}{T_{\gamma 0}^{2}},
\end{aligned}
$$

where $T_{\gamma 0}$ is the photon temperature when the neutron capture is accomplished. The final abundance is given by

$$
X_{f}=\exp (-t / \tau) \bar{X}
$$

and the final helium abundance by weight is

$$
Y_{4}=2 X_{f}
$$

Applying all these steps to the standard model, it results $Y_{4} \sim 0.243$. Note that this result is not exactly the same found in [10], which is $Y_{4} \sim 0.247$. We attribute this small discrepancy to the fact that we used a Mathematica program instead of a pocket calculator. Moreover, it is point out in [10] that their results is a kind of upper limit using the semi-analytical method described there. A more precise computation, using numerical code, gives $Y_{4} \sim 0.241$ [15].

The above expressions resume briefly the main steps. More important, it has been shown explicitly where the value of the Planck's mass (consequently, the gravitational coupling value) appears. This will enable us to compute the variation of the helium abundance as function of $G_{R}$, and consequently as function of the Brans-Dicke parameter $\omega$.

In [10], it was computed the variation on the helium abundance due to variations of the chemical potential of the electron neutrino $\mu$, the number of neutrinos $N_{\nu}$, 
the neutron time-life $\tau$ and ratio of photons to baryons $\eta$. They find

$$
\Delta Y_{4}=-0.25 \mu+0.014 \Delta N_{\nu}+0.18 \frac{\Delta \tau}{\tau}+0.009 \ln \frac{\eta}{\eta_{0}}
$$

where $\eta_{0}=5 \times 10^{-10}$ is the adopted value for the ratio of baryons to photons. One of our goals is to add to this expression the term concerning the variation of the gravitational coupling as a perturbation around its value computed using the value of $G$ today.

\section{Helium abundance varying $G$}

Now we turn to the computation of the helium abundance with the cosmological model developed in section II. First of all, we notice that, since in the radiative phase the scalar field is constant, all development exhibits in the previous section is valid; we must just to compute the value of the gravitational coupling (Planck's mass) in view of the fact that in the later dust phase the gravitational coupling varies with time. We fix in (10) $\rho_{m 0} \sim 10^{-29} \mathrm{~g} / \mathrm{cm}^{3}$ and $\rho_{r 0} \sim 0.950 \times 10^{-33} \mathrm{~g} / \mathrm{cm}^{3}$, which are approximately the densities of matter and radiation today [16]. Using $M_{P 0} \sim 1.221 \times 10^{19} \mathrm{Gev}$, we can then compute the values of $M_{P R}$ at the radiative phase in terms of $\omega$. With the value of $M_{P R}$, we can compute the helium mass fraction. The values are the following:

\begin{tabular}{||r||r||r||}
\hline \hline$\omega$ & $M_{P R}($ in $\mathrm{GeV})$ & $Y_{4}$ \\
\hline \hline-1 & $\infty$ & 1 \\
\hline-0.5 & $1.160 \times 10^{15}$ & 0.961 \\
\hline 0 & $1.190 \times 10^{17}$ & 0.818 \\
\hline 1 & $1.205 \times 10^{18}$ & 0.612 \\
\hline 10 & $8.015 \times 10^{18}$ & 0.318 \\
\hline 50 & $1.115 \times 10^{19}$ & 0.259 \\
\hline 500 & $1.166 \times 10^{19}$ & 0.251 \\
\hline 1,000 & $1.210 \times 10^{19}$ & 0.244 \\
\hline 10,000 & $1.215 \times 10^{19}$ & 0.243 \\
\hline
\end{tabular}

From this table, we can see that all matter becomes composed of helium for $\omega=-1$ (the case where the Brans-Dicke cosmology coincides with the string cosmology at low energy level [17]), and it approaches the asymptotic value of the standard model as $\omega$ increases. This result is easy to understand. Since in the radiative phase the Friedmann equation (19) is valid, as $G$ increases the rate of the expansion of the Universe also increases. Hence, the temperature drops very quickly and the duration of the nucleosynthesis era becomes shorter. All effects described in the preceding section contribute to decrease the quantity of neutrons. If the duration of the nucleosynthesis era becomes smaller, than more neutrons survive to be captured in deuterium, forming helium later. When $\omega=-1$ the value of the gravitational coupling diverges, there is an instantaneous transition from the radiative phase to the dust phase, and all initial neutrons survive.

We may evaluate now how a small change in the value of the gravitational coupling affects the nucleosynthesis as an approximation to the standard model. This will permit us to establish constraints on the variation of $G$ by using the nucleosynthesis observational results. The value of $G$ affects essentially the parameters $b, t_{c}$ and $T_{\gamma 0}$. Writing $G=G_{0}+\Delta G$, $\left(M_{P}=M_{P 0}+\Delta M_{P}\right)$, and introducing this quantity in the computation steps described before, we find that the helium matter fraction varies as

$$
\Delta Y_{4}=0.088 \frac{\Delta G}{G_{0}}
$$

It is generally argued that the nucleosynthesis observational results coincides with the theoretical results by with a precision of $1-2 \%$. Hence

$$
\frac{\Delta G}{G_{0}} \sim 1 \rightarrow \frac{\dot{G}}{G} \sim 10^{-10} \text { years }^{-1}
$$

where we have, in the last step, divided the first expression by the age of the Universe, assumed to be $t_{U} \sim 2 \times 10^{10}$ years, to obtain an estimation of the fractional time variation of $G$. This agrees in order of magnitude with the estimations of the time variation of $G$ using other methods[14, 15, 18]. Of course, a more precise comparison with other experimental determinations of $\frac{\dot{G}}{G}$ is not possible due to the approximations made.

\section{Conclusions}

Primordial nucleosynthesis is considered as one of the most precise tests of the standard cosmological scenario. With it, the abundances of helium, deuterium and lithium, due to the primordial processes, are computed. Comparison with observations showed that the predicted abundances agree with the observed ones by some percents. This is an impressive result since the primordial nucleosynthesis ocurred in the first seconds of the existence of the Universe. This high precise cosmological test may be used to constrain the values of some input parameters, like the number of neutrinos and the ratio of baryons to photons.

In the present work, we have estimated the influence of a possible variation of the gravitational coupling $G$ in the predicted abundance of light elements. In order to be specific, a Brans-Dicke cosmological scenario was constructed, with a radiative phase identical to that of the standard model, followed by a dust phase. The gravitational coupling decreases during the dust phase, while it remains constant during the radiative phase. 
Hence, the value of $G$ at the moment of the nucleosynthesis is, in this model, higher than the usual one.

The results indicate that for a larger $G$, the abundance of helium is higher. This is due to the fact that all the process occuring during the nucleosynthesis contribute to decrease the number of neutrons. A larger value of $G$ leads to a faster expansion of the Universe, decreasing the duration of the nucleosynthesis era. Consequently, the final number of neutrons that will survive, forming later the helium, is higher. The precision of the measurements of the abundance of the primordial elements permits to estimate the possible variation of $G$. We found however, that window of allowed values is not larger than that one obtained by other kind of experiments.

In all computation, it was used the simplest powerlaw type solution of the Brans-Dicke theory for the radiative phase, which is identical to the standard model one. This simplify a lot the evaluation of the helium abundance. However, it is possible in principle to consider more complicated scenarios. In fact, for the radiative phase, the Brans-Dicke scalar-field can be written as

$$
\dot{\phi}=\frac{C}{a^{3}},
$$

where $C$ is a constant. If $C$ is positive, the gravitational coupling decreases with time during the radiative phase; if it is negative, the gravitational coupling increases with time. The case studied before corresponds to $C=0$. For $C \neq 0$, we have the following solution for the scale factor:

$$
a(\xi)=a_{0}[\xi-B]^{\frac{1-\sqrt{\frac{3}{8 \omega}}}{2}}[\xi+B]^{\frac{1+\sqrt{\frac{3}{8 \omega}}}{2}},
$$

where $B=\sqrt{\frac{\bar{\omega}}{6}} C, \bar{\omega}=\omega+\frac{3}{2}$ and $\xi$ is the conformal time

$$
\xi=\int \frac{d t}{a}
$$

It will be interesting to consider such more general model, including a possible increase of the gravitational coupling during the radiative era, since it can be a possible solution to the discrepancy of the baryonic density obtained from nucleosynthesis and from CMB anisotropy. This leads, however, to more important modifications in the preceding computation, since some basic equations, like (19) must be modified by including the contribution of the non-minimal coupled scalar field. We hope to present this more general analysis in the future.

Acknowledgements: We thank $\mathrm{CNPq}_{\text {(Brazil) for }}$ partial financial support.

\section{References}

[1] A.M. Boesgaard and G. Steigman, Ann. Rev. Astron. Astrophys. 23, 319 (1985)

[2] R.A. Malaney and G.J. Mathews, Phys. Rep. 229, 145 (1993).

[3] S. Buerles, K. M. Nollett and M.S. Turner, Big-bang nucleosynthesis predictions for precision cosmology, astro-ph/0010171.

[4] V.V. Smith and D.L. Lambert, Astrophys. J. 345, L75 (1989).

[5] K.A. Olive, Phys. Rep. 333, 389 (2000).

[6] A.H. Jaffe et al., Phys. Rev. Lett. 86, 3475 (2000).

[7] C. B. Netterfield et al., A measurement by BOOMERANG of multiplepeaks in the angular power spectrum of the cosmic microwave background, astro-ph/0104460.

[8] D.F. Torres, Phys. Lett. B359, 249 (1995).

[9] J.A. Casas, J. Garcia-Bellido and M. Quirós, Phys. Lett. B278, 94 (1992).

[10] J. Bernstein, L.S. Brown and G. Feinberg, Rev. Mod. Phys. 61, 25 (1989).

[11] C. Brans and R.H. Dicke, Phys. Rev. 124, 925 (1961).

[12] S. Weinberg, Gravitation and Cosmology, Wiley, New York (1972).

[13] R.E. Lopez and M.S. Turner, Phys. Rev. D59, 3502 (1999).

[14] I.I. Shapiro et al. Phys. Rev. Lett. 26, 27 (1971).

[15] V.B. Braginsky, C.M. Caves and K.S. Thorne, Phys. Rev. D15, 2047 (1977).

[16] C.W. Misner, K.S. Thorne and J.A. Wheeler, Gravitation, W.H. Freeman, San Francisco (1973)

[17] M. Gasperini, Elementary introduction to pre-big bang cosmologyand to the relic graviton background, hepth/9907067.

[18] C.M. Will, Theory and experiment in gravitational physics, Cambridge University Press, Cambridge (1993). 